\title{
Identification of HIV Dynamic System in the Case of Incomplete Experimental Data
}

\author{
P. Mathye*, I. Fedotov* and M. Shatalov*† \\ * Department of Mathematics and Statistics \\ Tshwane University of Technology, Pretoria, South Africa \\ Email: mathyeph@gmail.com; FedotovI@tut.ac.za; ShatalovM@tut.ac.za \\ ${ }^{\dagger}$ Manufacturing and Materials \\ Council for Scientific and Industrial Research (CSIR), Pretoria, South Africa
}

Received: 6 October 2015, accepted: 14 December 2015, published: 17 January 2016

\begin{abstract}
In this paper we apply an inverse method that estimates parameters of deterministic mathematical models to an HIV model. We consider the case where experimental data concerning the values of some variables is incomplete or unknown. The objective is to estimate the parameters and to restore the information concerning the behaviour of the incomplete data. The method is based on integrating both sides of equations of a dynamic system, and applying some minimization methods (for example least square method). Such an approach was first suggested in [7] and [8]. Analysis of the HIV model and a corresponding numerical example is presented.
\end{abstract}

Keywords-Inverse problem, least square methods, parameter estimation, HIV model, incomplete data.

\section{INTRODUCTION}

Several HIV vaccine models have been developed over the recent years (e.g., [1] and [4]). These models describe and predict the potential epidemiological impact of vaccination. For the models to give insight into the transmission dynamics of HIV, model parameters are of great significance. The parameters for these model are estimated based on HIV seroprevalence data. Most often the data on testing and treatment history is incomplete (missing). This barrier can be as a result of the stigma attached to and the discrimination against people living with HIV and AIDS. In studying the dynamics of this world pandemic, the availability of recorded valuable data is thus a challenge.

The proposed method is based on eliminating the unknown (missing) state variables from the original system algebraically. The resultant system is then used to estimate the unknown model parameters. The discussion on the identification of dynamic systems with incomplete data and restoring the missing data is elementary and can be suitable for students in basic courses on dynamic systems identification.

A four dimensional deterministic model for transmission dynamics of HIV in the presence of a preventive vaccine is considered as an example. The model is identified in the cases of incomplete data. It is assumed that the population sizes of individuals infected by the wild type strain and by both the wild and the vaccine strains is unknown.

For this study, the data is artificially generated from the given parameters from Gumel's paper [4]. We then 'forget' about the these parameters and 
part of the generated data. Applying the proposed method we estimate the parameters and then restore the 'forgotten' data. The performance of the proposed method with real life data remains to be investigated in the future.

The remainder of the paper is structured as follows. Section II briefly outlines the formulation of the problem. Section III discusses the inverse method used in solving the problem. Section IV gives a description of the mathematical model that is used as an example. Sections V, VI and VII discusses the method of solution, parameter estimations and a numerical simulation respectively. Finally, concluding remarks are made in Section VIII.

\section{PROBLEM FORMULATION}

Consider a system of ordinary differential equations of the form

$$
\begin{aligned}
& \frac{d x}{d t}=f(t, x, y, \theta), \\
& \frac{d y}{d t}=g(t, x, y, \theta),
\end{aligned}
$$

where $(x, y)$ is a vector-function $[0, T] \ni t \rightarrow(x, y) \in \mathbf{R}^{m} \times \mathbf{R}^{n}$ subject to experimental information concerning the values of $x\left(t_{j}\right)$ at points $t_{j} \in[0, T],\left(t_{j}=0,1, \ldots, N\right)$ are known. Now, suppose the information concerning the values $y\left(t_{j}\right)$ is either incomplete or unknown. It may be, for example, some statistical data of the form:

Table 1: Experimental data.

\begin{tabular}{|l|l|l|l|l|}
\hline$t_{0}$ & $\cdots$ & $t_{j}$ & $\cdots$ & $t_{N}$ \\
\hline $\mathbf{x}_{0}=\mathbf{x}\left(t_{0}\right)$ & $\cdots$ & $\mathbf{x}_{j}=\mathbf{x}\left(t_{j}\right)$ & $\cdots$ & $\mathbf{x}_{N}=\mathbf{x}\left(t_{N}\right)$ \\
\hline $\mathbf{y}_{0}=?$ & $\cdots$ & $\mathbf{y}_{j}=?$ & $\cdots$ & $\mathbf{y}_{N}=?$ \\
\hline
\end{tabular}

The parameter $\theta$ is $l$ dimensional, that is $\theta \in$ $\mathbf{A} \subset R^{l}$ where $\mathbf{A}$ can coincide with $R^{l}$ (no constrains between the entries of $\theta$ ) and $\mathbf{A}$ can be subset of $\mathbf{R}^{n}$ (there are constraints).

The purpose of this study is to identify the model parameters $\theta$ and to restore (recover) the information concerning the behaviour of the incomplete or unknown of $y(t)$.

\section{INVERSE METHOD}

Solve (1) with respect to y to get

$$
y=h\left(t, x, \frac{d x}{d t}, \theta\right) \text {. }
$$

Substitute $y$ by $h\left(t, x, \frac{d x}{d t}, \theta\right)$ in 2 to obtain

$\frac{d}{d t} h\left(t, x, \frac{d x}{d t}, \theta\right)=g\left(t, x, h\left(t, x, \frac{d x}{d t}, \theta\right), \theta\right)$.

For this study consider the case where (4) is linear with respect to the coefficients $c_{k}(\theta)$ :

$\frac{d}{d t} h_{0}\left(t, x, \frac{d x}{d t}\right)=\sum_{k=1}^{N} c_{k}(\theta) h_{k}\left(t, x, \frac{d x}{d t}, \frac{d^{2} x}{d t^{2}}\right)$

where $c_{k}: \mathbf{A} \ni \theta \mapsto c_{k}(\theta)$ is a scalar function.

The parameter identification for the model is based on the direct integration of the dynamic system with posterior application of a quadrature rule (for example, the adaptive trapezoidal rule). Some minimization methods (for example, least squares method (see for example [5])) is then applied to find the estimates.

Integrating (5) twice with respect to $t$ from $t_{0}$ to $t_{i},(i=1,2, \ldots, N)$ yields:

$$
\mathbb{A} \alpha-\mathbf{h}=0
$$

where

$$
\begin{aligned}
& \mathbb{A}=\int_{t_{0}}^{t_{i}}\left(t_{i}-\tau\right) h_{k}\left(\tau, x(\tau), \frac{d x(\tau)}{d \tau}, \frac{d^{2} x(\tau)}{d \tau^{2}}\right) d \tau \\
& \alpha=c_{k}(\theta), \text { and } \mathbf{h}=\int_{t_{0}}^{t_{i}} h_{0}\left(\tau, x(\tau), \frac{d x(\tau)}{d \tau}\right) d \tau
\end{aligned}
$$

The values of the unknown parameters $\alpha$ in $(6)$ can be determined using method of least squares.

Suppose there are some constraints to be satisfied amongst the parameters $\alpha$. The problem is thus to minimize the Lagrangian

$$
\begin{aligned}
L(\alpha, \lambda)=(\mathbb{A} \alpha-\mathbf{h})^{\top} & (\mathbb{A} \alpha-\mathbf{h}) \\
& +2 \lambda^{\top}(\mathbf{C} \alpha-\mathbf{b}),
\end{aligned}
$$

where $\lambda$ is the Lagrange multiplier and

$$
\mathbf{C} \alpha=\mathbf{b}
$$


the constraints that must be satisfied.

The necessary conditions for a constrained minimum at $\alpha^{*}$ is the existence of vector $\lambda^{*}$ such that

$\nabla_{\alpha} L\left(\alpha^{*}, \lambda^{*}\right)=\left(\alpha^{*}\right)^{\top} \mathbb{A}^{\top} \mathbb{A}-\mathbf{h}^{\top} \mathbb{A}+\left(\lambda^{*}\right)^{\top} \mathbf{C}=0$

and

$$
\nabla_{\lambda} L\left(\alpha^{*}, \lambda^{*}\right)=\mathbf{C} \alpha^{*}-\mathbf{b}=0
$$

That is, we obtain the system:

$$
\left(\begin{array}{cc}
\mathbb{A}^{\top} \mathbb{A} & \mathbf{C}^{\top} \\
\mathbf{C} & 0
\end{array}\right)\left(\begin{array}{l}
\alpha^{*} \\
\lambda^{*}
\end{array}\right)=\left(\begin{array}{c}
\mathbb{A} \\
\mathbf{b}
\end{array}\right)
$$

where the matrix:

$$
\left(\begin{array}{cc}
\mathbb{A}^{\top} \mathbb{A} & \mathbf{C}^{\top} \\
\mathbf{C} & 0
\end{array}\right)
$$

is a square matrix with a non-zero determinant. The solution to the linear system 111 is given by

$$
\left(\begin{array}{l}
\alpha^{*} \\
\lambda^{*}
\end{array}\right)=\left(\begin{array}{cc}
\mathbb{A}^{\top} \mathbb{A} & \mathbf{C}^{\top} \\
\mathbf{C} & 0
\end{array}\right)^{-1}\left(\begin{array}{l}
\mathbb{A} \\
\mathbf{b}
\end{array}\right)
$$

With the the parameters $\alpha=c_{k}(\theta)$ known, the information concerning the behaviour of the incomplete or unknown state variable $y(t)$ can now be restored from $(3)$.

\section{MAthematicAl MODEL}

The model monitors four populations namely: HIV susceptible $(X)$, unvaccinated individuals infected by wild type $\left(Y_{w}\right)$, individuals uninfected by the wild type but infected by the vaccine strain $\left(Y_{v}\right)$ and individuals dually-infected with the vaccine and wild strain $\left(Y_{v w}\right)$. The total (sexual activity) population size is $N=N(t)=X+$ $Y_{v}+Y_{w}+Y_{v w}$. The model is a modified version of those studied by Blower and Gumel in [1] and [4] respectively.

\section{A. HIV susceptible $(X)$}

Individuals are recruited, by birth or immigration, into this population at the rate $p_{1}$. This population is reduced by the natural cessation of sexual activity at a rate $\mu$, infection with the vaccine strain at the rate $\alpha_{v}$, the wild strain and infected by the dual infected individuals at the rate $\alpha_{w}$. In this case it is assumed that the dual infected individuals can only transmit the wild strain. Thus,

$\frac{d X}{d t}=p_{1}-\mu_{1} X-\alpha_{v} \frac{X Y_{v}}{N}-\alpha_{w} \frac{X\left(Y_{w}+Y_{v w}\right)}{N}$

B. Individuals uninfected by the wild type but infected by the vaccine strain $\left(Y_{v}\right)$

This population increases through the new susceptible being vaccinated at the rate $p_{2}$ and by infection by the vaccine strain at the rate $\alpha_{v}$. It is decreased by infection with the wild strain and infected by the dual infected individuals at the rate $\gamma$. The parameter $\gamma$ is of the form $(1-\psi) c \beta_{w}$ where $\psi$ is the degree of protection that the vaccine provides against infection with the wild-type strain, $c$ the number of sexual partners and $\beta_{w}$ the rate of infection by the wild-type strain. The population is further decreased by natural cessation from sexual activity and by death induced by infection with the wild strain at a rate $\mu_{2}$.

$$
\frac{d Y_{v}}{d t}=p_{2}+\alpha_{v} \frac{X Y_{v}}{N}-\gamma \frac{Y_{v}\left(Y_{w}+Y_{v w}\right)}{N}-\mu_{2} Y_{v}
$$

\section{Unvaccinated individuals infected by wild type} $\left(Y_{w}\right)$

This population increases through the susceptible being infected by the wild strain and by the dual infected individuals at the rate $\alpha_{w}$. The population is decreased by natural cessation from sexual activity and by death induced by infection with the wild strain at a rate $\mu_{3}$.

$$
\frac{d Y_{w}}{d t}=\alpha_{w} \frac{X\left(Y_{w}+Y_{v w}\right)}{N}-\mu_{3} Y_{w},
$$

D. Individuals dually-infected with the vaccine and wild strain $\left(Y_{v w}\right)$

This population increases through infection with the wild strain and infected by the dual infected individuals at the rate $\gamma$. The population is decreased by natural cessation from sexual activity 
P. Mathye et al., Identification of HIV Dynamic System in The Case of Incomplete ...

and by death induced by infection with the wild strain at a rate $\mu_{4}$.

$$
\frac{d Y_{v w}}{d t}=\gamma \frac{Y_{v}\left(Y_{w}+Y_{v w}\right)}{N}-\mu_{4} Y_{v w}
$$

The complete model is thus:

$$
\left.\begin{array}{rl}
\frac{d X}{d t} & =p_{1}-\mu_{1} X-\alpha_{v} \frac{X Y_{v}}{N}-\alpha_{w} \frac{X\left(Y_{w}+Y_{v w}\right)}{N}, \\
\frac{d Y_{v}}{d t} & =p_{2}+\alpha_{v} \frac{X Y_{v}}{N}-\gamma \frac{Y_{v}\left(Y_{w}+Y_{v w}\right)}{N}-\mu_{2} Y_{v}, \\
\frac{d Y_{w}}{d t} & =\alpha_{w} \frac{X\left(Y_{w}+Y_{v w}\right)}{N}-\mu_{3} Y_{w}, \\
\frac{d Y_{v w}}{d t} & =\gamma \frac{Y_{v}\left(Y_{w}+Y_{v w}\right)}{N}-\mu_{4} Y_{v w},
\end{array}\right\}
$$

Adding all the equations of the system 18 gives

$$
\frac{d N}{d t}=p_{1}+p_{2}-\mu_{1} X-\mu_{2} Y_{v}-\mu_{3} Y_{w}-\mu_{4} Y_{v w}
$$

All model parameters are nonnegative.

\section{Method of Solution}

Suppose that $N=N(t), X=X(t), Y_{v}=Y_{v}(t)$ are known and that $Y_{w}$ and $Y_{v w}$ are unknown. From

$$
N=X+Y_{v}+Y_{w}+Y_{v w},
$$

it is clear that the sum $Y_{w}+Y_{v w}$ is also known. In fact,

$$
Y_{w}+Y_{v w}=N-X-Y_{v}
$$

Let

$$
Z=Y_{w}+Y_{v w}
$$

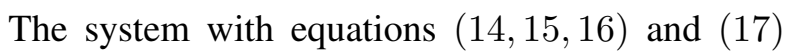
then becomes

$$
\begin{gathered}
\frac{d X}{d t}=p_{1}-\mu_{1} X-\alpha_{v} \frac{X Y_{v}}{N}-\alpha_{w} \frac{X Z}{N}, \\
\frac{d Y_{v}}{d t}=p_{2}+\alpha_{v} \frac{X Y_{v}}{N}-\gamma \frac{Y_{v} Z}{N}-\mu_{2} Y_{v}, \\
\frac{d Y_{w}}{d t}=\alpha_{w} \frac{X Z}{N}-\mu_{3} Y_{w} \\
\frac{d Y_{v w}}{d t}=\gamma \frac{Y_{v} Z}{N}-\mu_{4} Y_{v w} .
\end{gathered}
$$

By using the inverse method the parameters in Equations 23) and (24) can be determined. That is, parameters $p_{1}, p_{2}, \mu_{1}, \mu_{2}, \alpha_{v}, \alpha_{w}$ and $\gamma$ can now be considered known. Adding Equations (25) and (26) and rearranging yields

$$
\mu_{3} Y_{w}+\mu_{4} Y_{v w}=\alpha_{w} \frac{X Z}{N}+\gamma \frac{Y_{v} Z}{N}-\frac{d Z}{d t} .
$$

Let

$$
M=\alpha_{w} \frac{X Z}{N}+\gamma \frac{Y_{v} Z}{N}-\frac{d Z}{d t},
$$

Equation (27) then becomes

$$
\mu_{3} Y_{w}+\mu_{4} Y_{v w}=M .
$$

From $(22)$ and $(29)$, form the system

$$
\begin{gathered}
Y_{w}+Y_{v w}=Z, \\
\mu_{3} Y_{w}+\mu_{4} Y_{v w}=M .
\end{gathered}
$$

Solving this system we obtain

$$
Y_{w}=\frac{\mu_{4} Z-M}{\Delta}
$$

and

$$
Y_{v w}=\frac{M-\mu_{3} Z}{\Delta}
$$

where

$$
\Delta=\mu_{4}-\mu_{3} .
$$

Differentiating Equation 32 yields,

$$
\frac{d Y_{w}}{d t}=\frac{\mu_{4}}{\Delta} \frac{d Z}{d t}-\frac{1}{\Delta} \frac{d M}{d t}
$$

Equating (25) and 35 yields,

$$
\frac{\mu_{4}}{\Delta} \frac{d Z}{d t}-\frac{1}{\Delta} \frac{d M}{d t}=\alpha_{w} \frac{X Z}{N}-\mu_{3} Y_{w}
$$

Simplifying

$$
\frac{\mu_{4}}{\Delta} \frac{d Z}{d t}-\frac{1}{\Delta} \frac{d M}{d t}=\alpha_{w} \frac{X Z}{N}-\frac{\mu_{3} \mu_{4}}{\Delta} Z+\frac{\mu_{3}}{\Delta} M
$$

Rearranging gives,

$$
\frac{\mu_{4}}{\Delta} \frac{d Z}{d t}-\frac{1}{\Delta} \frac{d M}{d t}=\alpha_{w} \frac{X Z}{N}-\frac{\mu_{3} \mu_{4}}{\Delta} Z+\frac{\mu_{3}}{\Delta} M
$$

For convenience,

$$
a_{1} \frac{d Z}{d t}+a_{2} \frac{d M}{d t}+a_{3} M+a_{4} Z+F=0
$$


P. Mathye et al., Identification of HIV Dynamic System in The Case of Incomplete ...

where

$$
\begin{aligned}
& a_{1}=\frac{\mu_{4}}{\Delta}, a_{2}=-\frac{1}{\Delta}, a_{3}=-\frac{\mu_{3}}{\Delta}, \\
& a_{4}=\frac{\mu_{3} \mu_{4}}{\Delta} \text { and } F=-\alpha_{w} \frac{X Z}{N} .
\end{aligned}
$$

The relationship between the parameters $a_{1}, a_{2}, a_{3}$ and $a_{4}$ is given by the following constrains

$$
a_{1}+a_{3}=1
$$

and

$$
a_{1} a_{3}-a_{2} a_{4}=0
$$

Suppose that the parameters $a_{1}, a_{2}, a_{3}$ and $a_{4}$ are obtained, then

$$
\mu_{4}=-\frac{a_{1}}{a_{2}} \text { and } \mu_{3}=\frac{a_{1}-1}{a_{2}} .
$$

Finally, with Z, M, $\mu_{3}$ and $\mu_{4}$ known, the unknown variables $Y_{w}$ and $Y_{v w}$ can be restored from 32 and (33).

\section{Vi. Parameter Estimation}

Recall (23),

$$
\frac{d X}{d t}=p_{1}-\mu_{1} X-\alpha_{v} \frac{X Y_{v}}{N}-\alpha_{w} \frac{X Z}{N},
$$

Integrating both sides with respect to $t$ from $t_{0}$ to $t_{i}$, gives

$$
\Delta X_{i}=p_{1} t_{i}-\mu_{1} J_{i}-\alpha_{v} K_{i}-\alpha_{w} P_{i},
$$

where the integrals

$$
\Delta X_{i}=\int_{t_{0}}^{t_{i}} \frac{d X(\tau)}{d t} d \tau, K_{i}=\int_{t_{0}}^{t_{i}} \frac{X(\tau) Y_{v}(\tau)}{N(\tau)} d \tau
$$

$J_{i}=\int_{t_{0}}^{t_{i}} X(\tau) d \tau$ and $P_{i}=\int_{t_{0}}^{t_{i}} \frac{X(\tau) Z(\tau)}{N(\tau)} d \tau$,

are evaluated using the trapezoidal rules. The system 43 is of the form $\mathbb{A} \mathbf{a}=\mathbf{h}$, where

$$
\begin{aligned}
\mathbb{A} & =\left(\begin{array}{cccc}
t_{1} & -J_{1} & -K & -P_{1} \\
\vdots & \vdots & \vdots & \vdots \\
t_{N} & -J_{N} & -K & -P_{N}
\end{array}\right), \\
\mathbf{a} & =\left(\begin{array}{c}
p_{1} \\
\mu_{1} \\
\alpha_{v} \\
\alpha_{w}
\end{array}\right) \text { and } \mathbf{h}=\left(\begin{array}{c}
\Delta X_{1} \\
\vdots \\
\Delta X_{N}
\end{array}\right) .
\end{aligned}
$$

Solving the regression problem

$$
\operatorname{minimize}\|\mathbb{A} \mathbf{a}-\mathbf{h}\|_{2}
$$

using least squares method we obtain the estimate ã. Thus the parameters $p_{1}, \mu_{1}, \alpha_{v}$ and $\alpha_{w}$ are found.

Now recall 24,

$$
\frac{d Y_{v}}{d t}=p_{2}+\alpha_{v} \frac{X Y_{v}}{N}-\gamma \frac{Y_{v} Z}{N}-\mu_{2} Y_{v}
$$

Similarily, integrating both sides of 45 yields

$$
\Delta Y_{v}=p_{2} t_{i}+\alpha_{v} K_{i}-\gamma Q_{1}-\mu_{2} I_{i},
$$

where

$$
\begin{aligned}
& \Delta Y_{v}=\int_{t_{0}}^{t_{i}} \frac{d Y_{v}(\tau)}{d t} d \tau, K_{i}=\int_{t_{0}}^{t_{i}} \frac{X(\tau) Y_{v}(\tau)}{N(\tau)} d \tau, \\
& Q_{i}=\int_{t_{0}}^{t_{i}} \frac{X(\tau) Z(\tau)}{N(\tau)} d \tau \text { and } I_{i}=\int_{t_{0}}^{t_{i}} Y(\tau) d \tau .
\end{aligned}
$$

The system (46) is of the form $\mathbb{A} \mathbf{a}=\mathbf{h}$, where

$$
\begin{aligned}
& \mathbb{A}=\left(\begin{array}{ccc}
t_{1} & -Q & -I_{1} \\
\vdots & \vdots & \vdots \\
t_{N} & -Q & -I_{N}
\end{array}\right), \\
& \mathbf{a}=\left(\begin{array}{c}
p_{2} \\
\gamma \\
\mu_{2}
\end{array}\right) \text { and } \mathbf{h}=\left(\begin{array}{c}
\Delta Y_{1}-\alpha_{v} K_{1} \\
\vdots \\
\Delta Y_{N}-\alpha_{v} K_{N}
\end{array}\right) .
\end{aligned}
$$

Solving the regression problem

$$
\operatorname{minimize}\|\mathbb{A} \mathbf{a}-\mathbf{h}\|_{2}
$$

using least squares method we obtain the estimate . Thus the parameters $\gamma, \mu_{2}$ and $p_{2}$ are found.

Lastly recall (39),

$$
a_{1} \frac{d Z}{d t}+a_{2} \frac{d M}{d t}+a_{3} M+a_{4} Z+F=0
$$

subject to the constraints

$$
a_{1}+a_{3}-1=0 \text { and } a_{1} a_{3}-a_{2} a_{4}=0 .
$$

Integrating both sides of 48 with respect to $t$ from $t_{0}$ to $t_{i}$, gives

$$
a_{1} \Delta Z_{i}+a_{2} \Delta M_{i}+a_{3} U_{i}+a_{4} W_{i}+\Delta F_{i}=0
$$


where

$$
\begin{gathered}
\Delta Z_{i}=\int_{t_{0}}^{t_{i}} \frac{d Z(\tau)}{d t} \tau, \Delta M_{i}=\int_{t_{0}}^{t_{i}} \frac{d M(\tau)}{d t} \tau, \\
U_{i}=\int_{t_{0}}^{t_{i}} M(\tau) d \tau, W_{i}=\int_{t_{0}}^{t_{i}} Z(\tau) d \tau \text { and } \\
\Delta F_{i}=-\alpha_{w} \int_{t_{0}}^{t_{i}} \frac{X(\tau) Y_{v}(\tau)}{N(\tau)} d \tau .
\end{gathered}
$$

To find the unknown parameters $a_{1}, a_{2}, a_{3}$ and $a_{4}$, the Lagrangian $L_{3}$ can be written with one constraint as

$$
\begin{aligned}
L_{3}(\mathbf{a})= & \frac{1}{2} \sum_{i=0}^{n}\left[a_{1}\left(Z_{i}-U_{i}\right)+a_{2} M_{i}+a_{4} W_{i}+\right. \\
& \left.\left(\Delta F_{i}+U_{i}\right)\right]^{2}+\lambda\left(a_{1}-a_{1}^{2}-a_{2} a_{4}\right)
\end{aligned}
$$

where $\lambda_{1}$ is the Lagrange multipliers and $\mathbf{a}=$ $\left(a_{1}, a_{2}, a_{4}\right)$. To minimize the least-square functional $L_{3}$, set

$$
\frac{\partial L_{3}}{\partial a_{1}}=\frac{\partial L_{3}}{\partial a_{2}}=\frac{\partial L_{3}}{\partial a_{4}}=\frac{\partial L_{3}}{\partial \lambda}=0 .
$$

The resultant system of equations is then solved using the method proposed by Fedotov et al [3] for finding roots of transcendental algebraic equations. The parameter value $a_{3}$ is finally obtained from $a_{3}=1-a_{1}$.

\section{NUMERICAL SIMULATION}

In order to illustrate the effectiveness of the method a numerical example is presented. The system 18 is solved numerically by Adams method using a mathematical software Mathcad. The following parameter values and initial conditions from Gumel [4] are used.

$$
\begin{gathered}
p_{1}=400, p_{2}=1.6 \times 10^{3}, \alpha_{v}=2.5, \alpha_{w}=2.25, \\
\gamma=0.9 \mu_{1}=0.031, \mu_{2}=0.0331, \mu_{3}=0.0281, \\
\mu_{4}=0.231, X^{0}=8 \times 10^{4}, Y_{v}^{0}=2000, \\
Y_{w}^{0}=8000, Y_{v w}^{0}=8000
\end{gathered}
$$

The solution vectors obtained are taken as experimental data. We then assume that the experimental data concerning the state variables $Y_{w}, Y_{v w}$ and the model parameter values to be unknown. The method discussed in the sections above is then applied to estimate the model parameter values and restore the vectors $Y_{w}$ and $Y_{v w}$.

The results given in the table below, show a comparison of the actual parameters used, the estimated parameters and the percentage error given by $\|\alpha-\tilde{\alpha}\| /\|\alpha\| \times 100$.

Table 2: The parameter estimates and errors.

\begin{tabular}{cccc}
\hline Parameter & Actual value & Estimated value & \% Error \\
& $\alpha$ & $\tilde{\alpha}$ & \\
\hline$p_{1}$ & 400 & 400.142 & 0.035 \\
$p_{2}$ & $1.6 \times 10^{3}$ & $1.6 \times 10^{3}$ & 0.000 \\
$\mu_{1}$ & 0.031 & 0.031 & 0.000 \\
$\mu_{2}$ & 0.331 & 0.326 & 1.511 \\
$\mu_{3}$ & 0.281 & 0.282 & 0.356 \\
$\mu_{4}$ & 0.231 & 0.229 & 0.866 \\
$\alpha_{v}$ & 2.5 & 2.495 & 0.200 \\
$\alpha_{w}$ & 2.25 & 2.25 & 0.000 \\
$\gamma$ & 0.9 & 0.905 & 0.556 \\
\hline
\end{tabular}

From Table 2 it can be seen that the estimate parameters are close enough to the actual ones. The percentage relative errors for this estimates are mostly low than $1 \%$.

Let $Y_{w}:=\left(U^{<3>}\right)_{i}$ and $\left(Y_{v w}:=U^{<4>}\right)_{i}$ be the solution vectors obtained from solving the system (18) and $Y Y_{w_{i}}$ and $Y Y_{v w_{i}}$ the estimated vectors. From Figures 1 and 2, it can be seen that the estimated vectors, $Y Y_{w_{i}}$ and $Y Y_{v w_{i}}$ are within acceptable limit of error.

\section{CONCLUSION}

In this paper a method to identify dynamic mathematical models with incomplete (missing) data was discussed. The method was applied to a four dimensional HIV vaccination model. The model parameters and the unknown (missing) data were restored.

The proposed method gives a direct hint of what is necessary to measure in practice and what data can be analytically restored (found). 

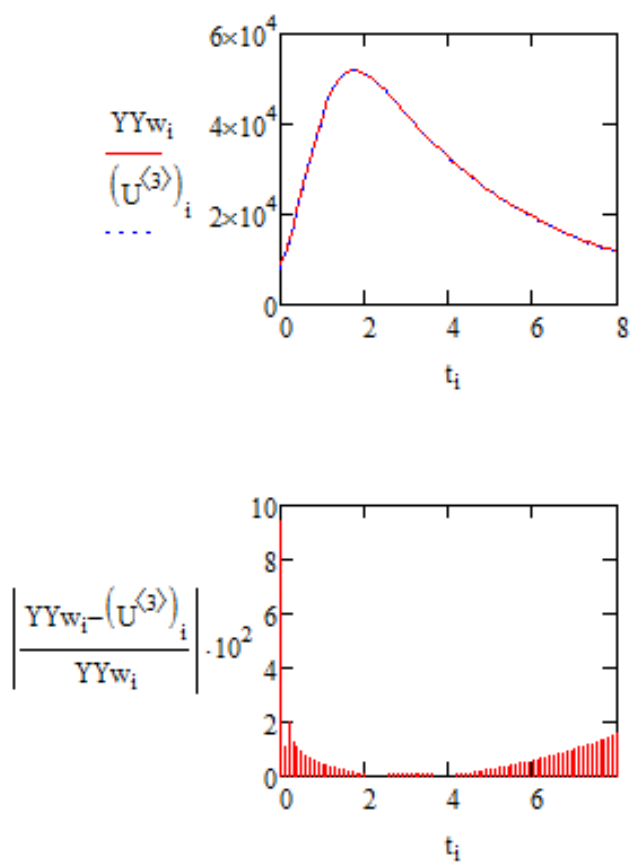

Fig. 1. Comparison of the estimated solutions, $Y Y w_{i}$, with the "experimental data", $U_{i}^{<3>}$, and the percentage relative error.

\section{REFERENCES}

[1] S. M. Blower, K. Koelle, D. Krischner \& J. Mills, Live attenuated HIV vaccine: predicting the trade-off between efficacy and safety, Proc Nat Acad Sci, 98(6) : $3618-$ 3623, 2001.

[2] F. Ding, G. Liu \& X. P. Liu, Parameter estimation with scarce measurements, Automatica, 47 : $1646-$ 1655, 2001.

[3] I. Fedotov, M. Shatalov \& J. N. Mwambakana, Roots of transcedental algebraic equations: A method of bracketing roots and selecting initial estimations, Buffelsfontein TIME 2008 Peer-review Conference Procedings, $22-26$ September, 2008.

[4] A. B. Gumel, S. M. Moghadas \& R. E. Mickens, Effect of a preventive vaccine on the dynamics of HIV transmission, Communication in Nonlinear Science and Numerical Simulations, 9 : 649 - 659, 2004.
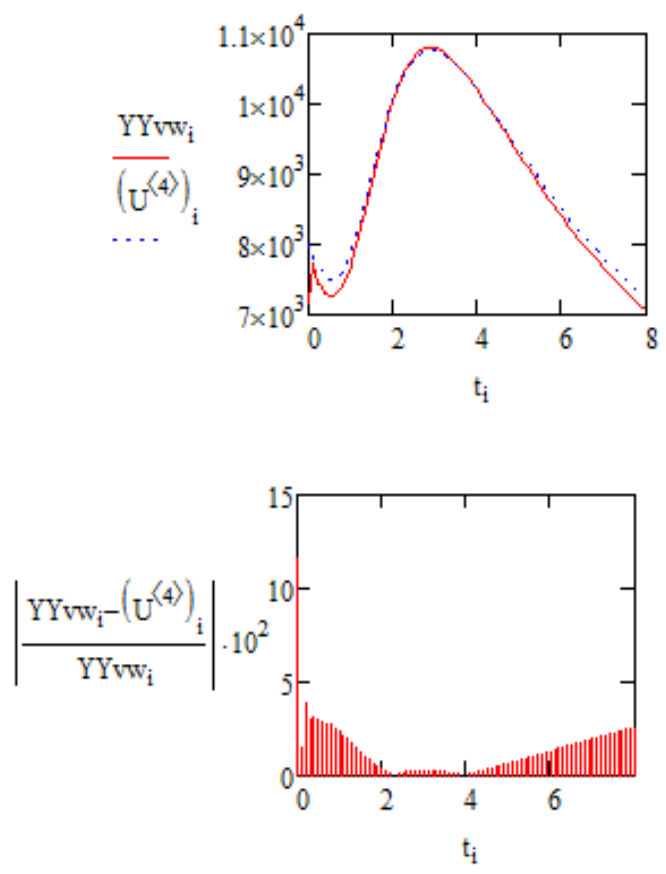

Fig. 2. Comparison of the estimated solutions, $Y Y v w_{i}$, with the "experimental data", $U_{i}^{<4>}$, and the percentage relative error.

[5] C. L. Lawson \& R. J. Hanson, Solving Least Square Problems, New Jersey, America: Prentice-Hall, 1974.

[6] M. A. Nowak\& A. R. Mclean, A mathematical model of vaccination against HIV to prevent the development of AIDS, RGMIA, Victoria University, 10(1,2) : 106-116, 2007.

[7] M. Shatalov \& I. Fedotov, On identification of dynamic systems parameters from experimental data, RGMIA, Victoria University, 10(1,2) : $106-116,2007$.

[8] M. Shatalov,I. Fedotov \& S. V. Joubert, A novel method of interpolation and extrapolation of functions by a linear initial value problem, Buffelsfontein TIME 2008 Peerreview Conference Procedings, 22 - 26 September, 2008. 